See discussions, stats, and author profiles for this publication at: https://www.researchgate.net/publication/339617985

\title{
A cohesion-controlled granular material
}

Preprint $\cdot$ March 2020

3 authors, including:

20.

Maxime Nicolas

Aix-Marseille Université

36 PUBLICATIONS 1,071 CITATIONS

SEE PROFILE

Some of the authors of this publication are also working on these related projects:

Project Cohesive powders View project

Project Sismiblock View project 


\title{
A cohesion-controlled granular material
}

\author{
Adrien Gans, ${ }^{*}$ Olivier Pouliquen, ${ }^{\dagger}$ and Maxime Nicolas ${ }^{\ddagger}$ \\ Aix Marseille Univ, CNRS, IUSTI, Marseille, France
}

(Dated: Accepted for publication in Physical Review E on February 20, 2020)

\begin{abstract}
We present a simple method to prepare a granular material with a controlled cohesion between particles. The granular material is made of spherical glass beads coated with a polyborosiloxane polymer. This material is proved to be stable in time and non-sensitive to temperature and humidity. The inter-particle force is measured and related to the size of the grain and the polymer coating thickness. Classical measurements (packing fraction, repose angle, macroscopic cohesion), are performed with this cohesion-controlled granular material. This model material opens many perspectives to study in a controlled manner the flow of cohesive grains.
\end{abstract}

\section{INTRODUCTION}

Handling powders and cohesive materials is an everyday challenge in many industrial processes. Beyond the difficulty of handling a very large number of particles, the cohesion between grains is a serious source of difficulty. Cohesion often comes into play when manipulating small particles, for which humidity $[1,2]$ or attractive forces like Van der Walls [3] or electrostatic forces [4], become important. The adhesion between the grains decreases the ability of powders to flow easily, and the dynamics then differs from dry granular materials made of large particles interacting solely trough frictional dry contact interactions. Whereas our knowledge of granular flows has improved over the last 20 years, the behavior of powders and cohesive granular media still represents a real challenge.

From an industrial point of view, different techniques have been developed to characterize and to quantify the "flowability" of powders $[5,6]$. This concept based on a compilation of different measurements (compaction, repose angles,...), although very useful when checking the quality of a product or when comparing different materials, lacks clear physical bases.

From a fundamental point of view, one difficulty in studying cohesive material is the control of the cohesion forces between the particles. Whereas in numerical studies, simplified interaction laws might be introduced in DEM approaches to simulated cohesive grains [7], experimentally, the control of the cohesion is more challenging. Working with actual powders faces many difficulties. Powder particles are often very polydispersed with a great variety of shape and surface roughness. A humidity and temperature control of the ambient air is required to ensure a good reproducibility of the experiments $[8,9]$. While some comparative tests between benchmark powders (such as lactose) are useful [10], it is still very difficult to link the particle properties to the bulk flow behavior of the powder [3], mainly because of a lack of a

\footnotetext{
* adrien.gans@univ-amu.fr

$\dagger$ olivier.pouliquen@univ-amu.fr

‡ maxime.nicolas@univ-amu.fr
}

controlled particle interaction. Most of the recent experimental works on cohesive granular materials have focused on capillary cohesion, studying the properties of an assembly of beads mixed with a small amount of a viscous liquid. The influence of the amount of liquid on the packing fraction $[11,12]$, on the repose or avalanche angle [1317], on the stability of a column [18] has been studied. The strength of a wet granular assembly has been trialed through the measurement of the shear modulus [19] and more recently, rheology experiments have been also conducted [20, 21] in a pressure imposed rheometer for glass beads coated by viscous silicon oil. Despite a good knowledge of the cohesion force arising from capillary bridges [22] the bulk behavior and the rheological measurements suggest a complex dynamics due to the evolution of the capillary bridges during shear, which may migrate and coalesce [23, 24]. Recently [25], silanization has been used to modify the surface properties of glass particles and build a cohesive force of $0.1 \mathrm{mN}$ order of magnitude. Experimental research on cohesive materials would strongly benefit from a model system making it possible to tune at will, and through a simple process, the cohesion between the grains.

In this paper we present a new method to prepare a controlled-cohesion granular material (CCGM) made of polyborosiloxane (PBS) coated glass particles, which suits many of the requirements to achieve experiments with a controlled cohesion. The main point is that the cohesion force between particles can be easily tuned through the PBS coating. The coating of the particles is easy and does not require heavy chemical equipment. Furthermore, the CCGM is very stable on a long time scale, is insensitive to humidity of the ambient air, and is also insensitive to room temperature.

The preparation method is first presented in Sec. II. A detailed studied of the inter-particle cohesive force induced by the presence of the PBS coating is presented in Sec. III. In Sec. IV the CCGM is tested in different classical configurations used for characterizing granular media : measurements of the bulk density, pile angle, inclined plane experiments. Concluding remarks and perspectives are given in Sec. V. 


\section{DESIGN OF A COHESION CONTROLLED GRANULAR MATERIAL}

The particle coating material is a polyborosiloxane (PBS) made from a -OH terminated Polydimethylsiloxane (PDMS) cross-linked with boric acid $\left(\mathrm{H}_{3} \mathrm{BO}_{3}\right)[26$, 27]. Each batch of CCGM is prepared with a mass $m_{g}$ of spherical glass beads (diameter $d$ and density $\rho_{G}=2600$ $\mathrm{kg} \cdot \mathrm{m}^{-3}$ ) with a small polydispersity, a mass $m_{P}$ of PDMS (density $\rho_{P}=970 \mathrm{~kg} \cdot \mathrm{m}^{-3}$, viscosity $750 \mathrm{mPa} \cdot \mathrm{s}$ and gyration radius $r_{g}=17 \AA$ ), and a mass $m_{A}$ of boric acid. We kept a constant mass ratio of boric acid over PDMS $m_{A} / m_{P}=0.14$. The boric acid is first dissolved in a small volume $(50 \mathrm{ml})$ of purified water heated at $60^{\circ} \mathrm{C}$. The particles, the PDMS and the $\mathrm{H}_{3} \mathrm{BO}_{3}$ solution are then mixed together in a heating mixer (Kenwood Cooking Chef) at $110{ }^{\circ} \mathrm{C}$ during $90 \mathrm{~min}$ to ensure the evaporation of the water and a homogeneous PBS coating of the spherical particles. After cooling, the batch is ready to use for experiments. The PBS has been characterized in a rheometer using a plane-plane geometry and the real and imaginary part of the shear modulus are $G^{\prime}=35 \mathrm{kPa}$ and $G^{\prime \prime}=7.5 \mathrm{kPa}$ with a relaxation time constant of $3.8 \mathrm{~s}$.

The range of particle size was $d=[0.8-10] \mathrm{mm}$ in experiments of Sec. III concerning the measurement of the inter-particle force, and $d=[0.2-1.4] \mathrm{mm}$ in the macroscopic experiments of Sec. IV. From atomic force microscopy imaging, the average roughness of $0.8 \mathrm{~mm}$ particles was $30 \pm 2 \mathrm{~nm}$. The effect of the PBS coating is qualitatively illustrated in Fig. 1 where a sample of $3 \mathrm{~mm}$ diameter coated glass beads reveals the cohesive nature of the material. The parameter controlling the cohesion in our study is the averaged thickness $b$. Assuming an homogeneous coating of a $\Omega_{P}=m_{P} / \rho_{P}$ volume of PBS over perfectly spherical particles of volume $\Omega_{G}=m_{G} / \rho_{G}$ gives an average thickness

$$
b=\frac{d}{2}\left[\left(1+\frac{\Omega_{P}}{\Omega_{G}}\right)^{1 / 3}-1\right]
$$

In the limit of a small coating averaged thickness $(b \ll d)$, the PBS volume ratio $\Omega_{P} / \Omega_{G} \approx 6 b / d$.

An attempt to visualize the PBS layer is presented in Fig. 2. On the pictures, the PBS appears as a grey and foamy fluid, which is optically different from the clean glass surface. The pictures of the clean surfaces have been obtained after a careful removal of the PBS with a spray of heptane without touching or moving the particle. The iridescence seen in Fig. 2(b) indicates that the PBS layer is not perfectly uniform and may suffer from thickness variation, and that some "holes" in the coating layer may also exist (Fig. 2a). However, a statistical analysis of the inter-particle force discussed later in the paper (see Sec. III) shows that the presence of defects weakly affects the cohesion between two particles. Since the coating layer is very thin, no capillary structure has been observed in optical microscopy when putting two

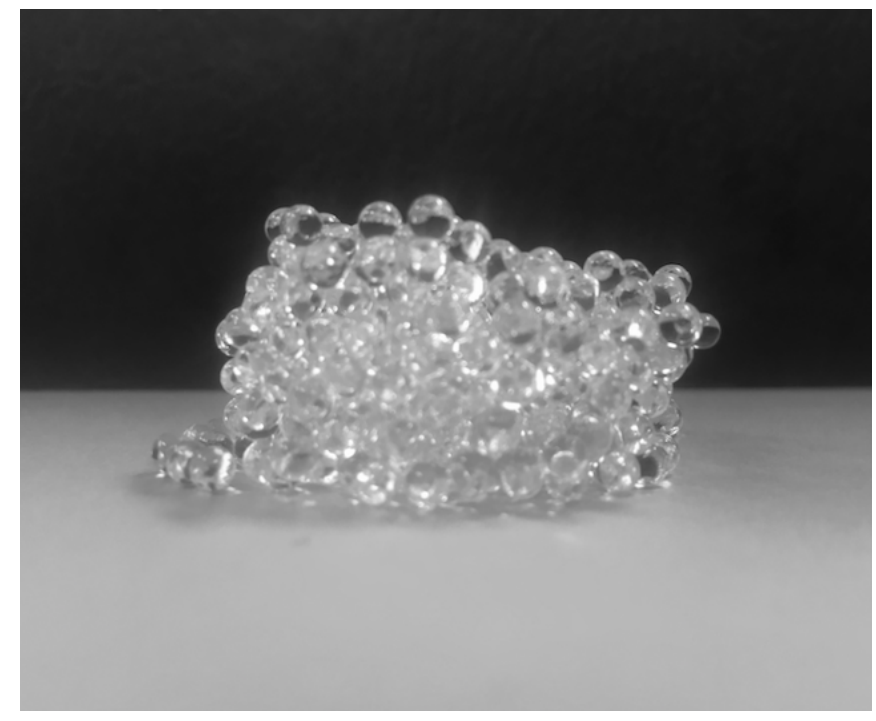

FIG. 1. Example of a cohesion-controlled granular material: a pile of glass beads $d=3 \mathrm{~mm}$ with a PBS coating layer of thickness $b=2.2 \mu \mathrm{m}$
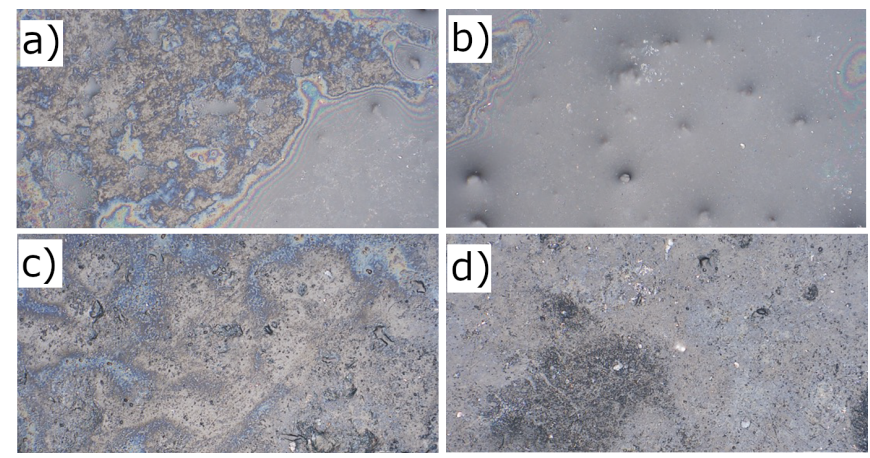

FIG. 2. Close-up visualization of the PBS coating on two different sample glass beads $(d=10 \mathrm{~mm})$ with an optical microscope (magnification $\times 700$ ). (a) A well coated area of particle 1 and (c) the same area after cleaning with a spray of heptane. (b) An irregularly coated area of particle 2 and (d) the same clean area. Iridescence can be seen where the PBS layer is not homogeneous.

beads in contact.

A last important remark is that the strong Si-OH link between the polymer and the glass bead surface helps the PBS to stick permanently on the particles. No drainage of the fluid was observed even for very long time (on the timescale of a year), making the material very stable in time, as will be discussed in section Sec. IV.

\section{INTER-PARTICLE COHESION FORCE MEASUREMENTS}

The cohesion force between two particles is a key parameter to control the macroscopic cohesion of a granular 
assembly. In this section we present the results of different experiments designed to measure the contact force between two particles due to the PBS coating. As presented in the previous section, the control parameters are: the size of the beads (diameter $d$ ), the PBS averaged layer thickness $b$, and the duration of the contact. We designed two methods to accurately measure the cohesion force. The first method uses the torque-meter of a commercial rheometer (MCR501 Anton Paar). It provides accurate measurements but performing a statistical analysis is tedious to. The second method is a home-designed force measurement device allowing simultaneous measurements on 10 pairs of particles.

\section{A. Role of the pre-compression load}

The cohesion force between two CCGM particles has been first measured using the rotating head of a AntonParr MCR501 rheometer. A sketch of the experimental set-up is given in Fig. 3a. A coated particle is attached to a fixed rigid structure through a linear spring and a similar coated particle from the same batch is glued at the end of an arm attached to the rheometer head. The two particles were put in contact and a pre-compression torque $T_{p c}$ corresponding to a pre-compression force $F_{p c}$ was applied before slowing reversing the applied torque up to the point when the two particles suddenly detach. The critical torque when detachment occurs provides the measurement of the cohesion force $F_{c}$. (Fig. 3a).

We measured the cohesion force using $d=10 \mathrm{~mm}$ particles and with a coating $b=5 \mu \mathrm{m}$, and the precompression force $F_{p c}$ was varied from $0.08 \mathrm{~N}$ to $2 \mathrm{~N}$. Fig. 4 (a) shows that the cohesion force $F_{c}$ does not depend on the pre-compression force $F_{p c}$, and that the order of magnitude of the cohesion force is $F_{c} \approx 5 \mathrm{mN}$ (dashed line). This independence of the cohesion with the compression force has been also observed in a different system by Kobayashi et al. [28]. With this setup, we also studied if the cohesion force was affected by the number of successive contacts. One can wonder if the polymer layer can be altered after the first sticking contact. Fig. 4(b) shows for three different pre-compression forces that the cohesion force is independent of the number of successive contacts. We therefore conclude that the PBS layer is strongly attached to the glass bead surface and that the stick-pull process occurring for a binary contact is reversible. A last important remark is that variation in the mean cohesion force is observed in Fig. 4(b): the mean cohesion is $F_{c}=4.3 \mathrm{mN}$ for the triangle symbols and $F_{c}=3.7 \mathrm{mN}$ mean force for the star symbols. This is an indication that the cohesion force may vary from one pair of particle to another and that a statistical analysis is necessary. This has motivated us to develop a second experimental set-up to measure in parallel the cohesion force for 10 pairs of particles. (a)

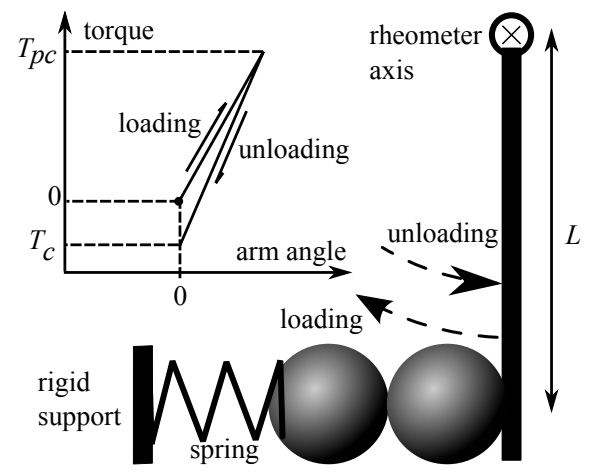

(b)

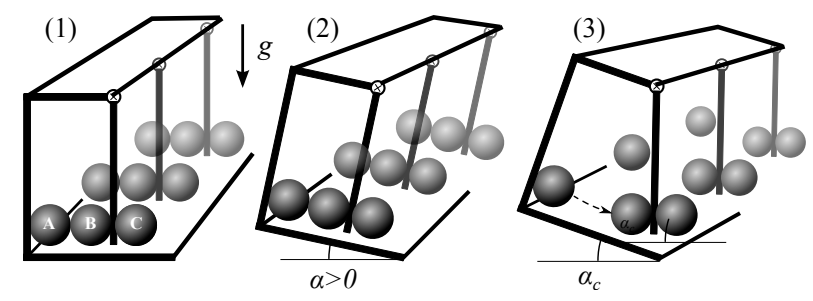

FIG. 3. (a) Sketch of the setup to measure the cohesion force for different pre-compression force using the rheometer torque-meter. The two particles are put in contact with a precompression force $F_{p c}=T_{p c} / L$, where $L=3.5 \mathrm{~cm}$ is the arm length and the cohesion force $F_{c}=T_{c} / L$ is measured when the two particles detaches. (b) Sketch of the pendulum experimental setup. Particle $\mathrm{A}$ is attached to a rigid structure, particle $\mathrm{B}$ and $\mathrm{C}$ are attached to the two sides of a pendulum. $F_{c}$ is measured by inclining the setup. 10 pendulums were mounted in parallel.

\section{B. Role of the contact waiting time}

The second home-made force measurement device is sketched in Fig. $3 \mathrm{~b}$ and consists in a set of 10 independent parallel pendulums. Each pendulum had one particle (B) attached at the bottom of the arm (Fig. 3b), which came into contact with a fixed particle (A). A third particle (C) was also glued on the other side of the pendulum arm and played the role of a counter-weight. The setup was mounted on a table that can be inclined. Starting from a nearly horizontal position (step 1 in Fig. 3, the table was slowly inclined with a rate $10^{\circ} \cdot \mathrm{min}^{-1}$ (step 2) until all the pairs of particles detached (step 3). The whole measurement process was recorded with a camera, and each time a pair of particles was detaching, the angle $\alpha_{c}$ was recorded and the cohesion force $F_{c}$ was computed from the torque balance.

With this device, the influence of the contact waiting time between two coated particles has been investigated from $5 \mathrm{~s}$ to 2 hours. We also investigated a 24 hours waiting time but the results were not significantly different from the 2 hours result. Fig. 5 shows that the cohesion force varies with contact time $t_{c}$ for $t_{c} \leqslant 600 \mathrm{~s}$ ) but eventually saturates for long contact times $t_{c}>1000$ s. This confirms the qualitative observations made when 

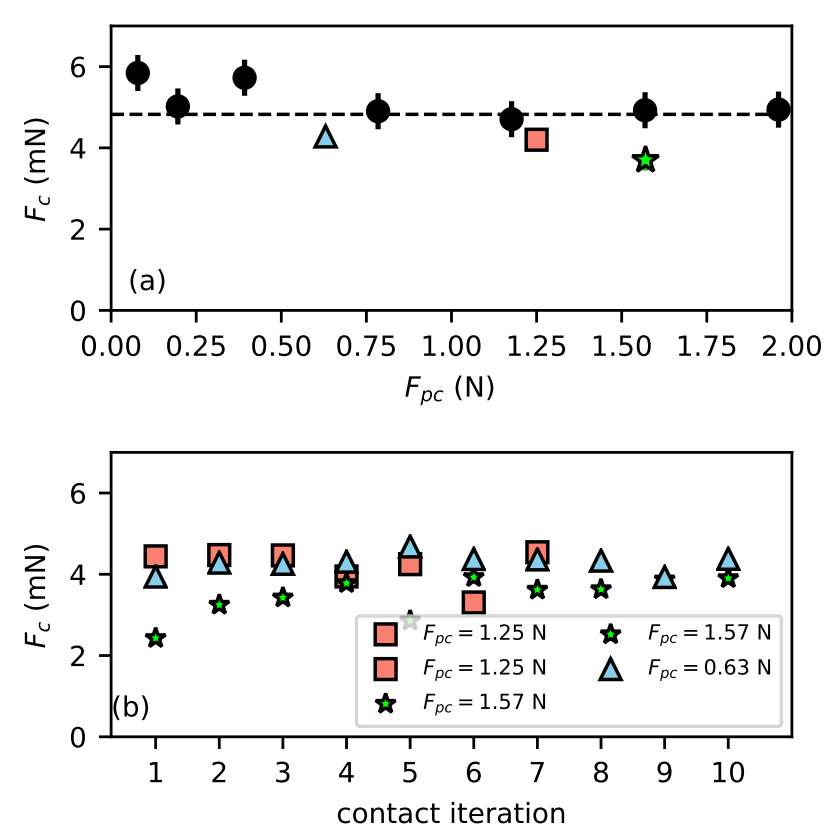

FIG. 4. (a) Cohesion force measured for different precompression forces $d=10 \mathrm{~mm}, b=5 \mu \mathrm{m}$. The dashed line indicates the mean cohesion force. Empty coloured symbols refer to the legend of (b). (b) Cohesion force for successive contacts, and for different pre-compression forces. The contact waiting time was kept constant equal to 10 minutes. If not visible, the error bars are smaller than the symbol size.

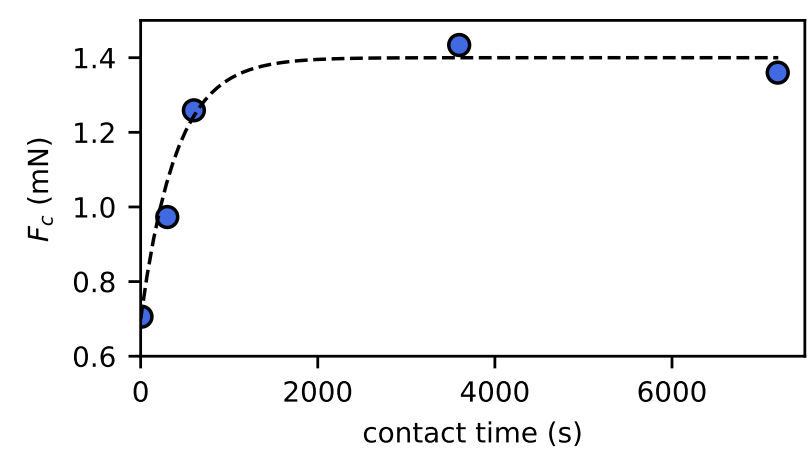

FIG. 5. Cohesion force as a function of the duration of the contact. The dashed line is a qualitative trend illustrating an exponential relaxation with time.

handling the CCGM out of storage. A CCGM stored during a long time looks more cohesive, although a vigorous shaking of the packing which renews all the contacts seems to diminish the cohesive nature of the sample. In the following, we now refer to "short" waiting time experiments when $t_{c}=10 \mathrm{~s}$, and "long" waiting time experiments when $t_{c}=10 \mathrm{~min}$.

The cohesion force distribution has been measured for a hundred pairs of particles out of the same batch $(d=5$

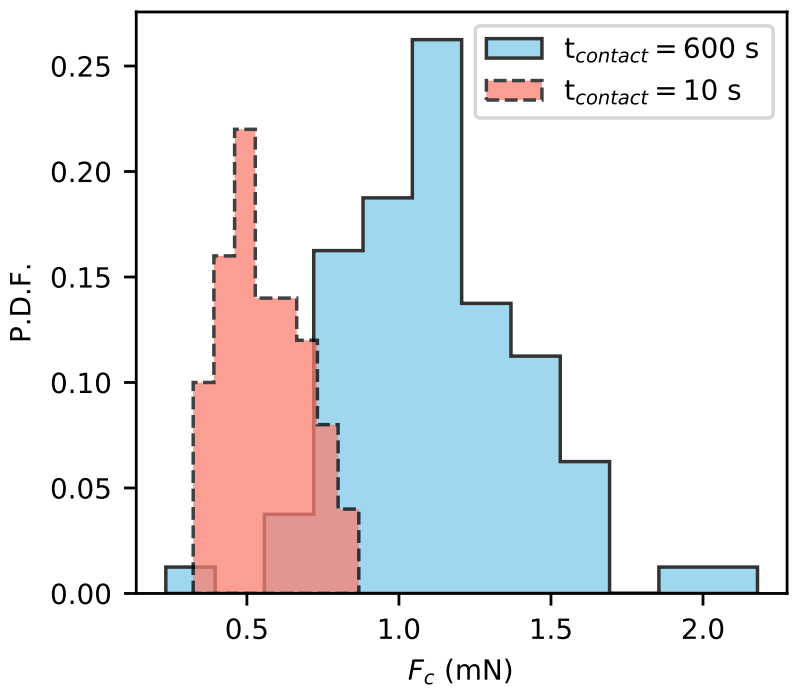

FIG. 6. Probability distribution function of the cohesion force measured for approximately 100 pairs of particles, for two different contact times $\left(t_{c}=10 \mathrm{~s}\right.$ and $\left.t_{c}=10 \mathrm{~min}\right)$ and for $d=5 \mathrm{~mm}, b=2 \mu \mathrm{m}$ coated particles.

$\mathrm{mm}, b=2 \mu \mathrm{m})$. The probability distribution function $p\left(F_{c}\right)$ is shown in Fig. 6 for short and long contact time. The averaged cohesion force is $0.56 \pm 0.1 \mathrm{mN}$ and $1.14 \pm 0.3$ $\mathrm{mN}$ for short and long contact time respectively. For $t_{c}=10 \mathrm{~s}$ the cohesion force distribution is narrower than for $t_{c}=10 \mathrm{~min}$. We have not investigated in more details the influence of the contact time and the origin of the force distribution, which are certainly related to the coating property of the particle and to the entanglement dynamics of the polymer chains.

\section{Scaling of the cohesion force}

To understand the physical origin of the cohesion force, we have systematically studied how it varies with the particle diameter $d$ and the averaged PBS coating thickness $b$. In Fig. 7, the cohesion force is plotted as a function of the particle diameter $d$ for a constant layer thickness $b=2 \mu \mathrm{m}$, and for the short and long waiting contact times. In the range $0.8<d<7 \mathrm{~mm}$ the cohesion force increases with the particle diameter. The cohesion force varies linearly with the diameter for short contact time but exhibits a more rapid increase for long contact time. The linear variation can be well described by a capillary model at contact [29]

$$
F_{c}=\frac{3}{2} \pi \gamma d,
$$

where the surface tension $\gamma \approx 24 \mathrm{mN} \cdot \mathrm{m}^{-1}$, a relevant order of magnitude for PDMS. For long contact times, other molecular phenomena may occur, such as a slow 


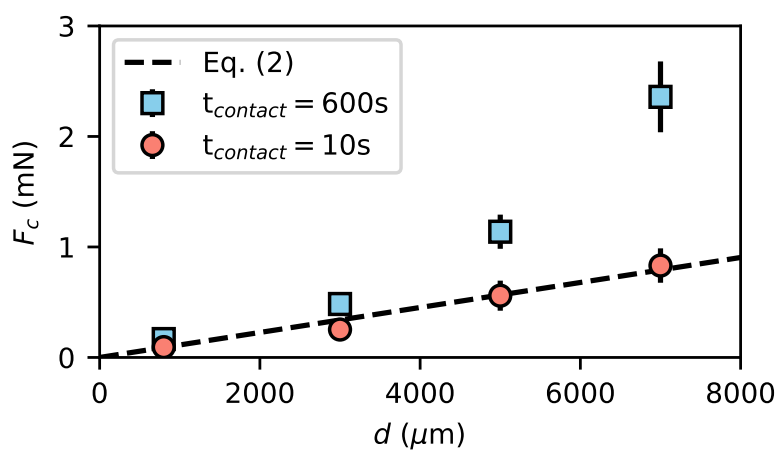

FIG. 7. The cohesion force $F_{c}$ as a function of the particle diameter $d$ for short (10 s) (circles) and long (10min) (squares) contact times. The dashed line is the linear expression (2).

polymer entanglement between PDMS polymers and we did not investigate further the long time correlation between the cohesion force and the particle radius.

The influence of the coating PBS thickness $b$ on $F_{c}$ is studied in Fig. 8 for different particle diameters $d$. The cohesion force normalized by the expression (2) is plotted as a function of $b$. We first observe that all the data obtained for different particle diameters collapse on a single curve. The normalized cohesion force starts from zero when there is no coating, increases and reaches a plateau equal to 1 when the averaged thickness of PBS is larger than $1 \mu \mathrm{m}$. An ad-hoc expression for the cohesion force can be proposed:

$$
F_{c}=\frac{3}{2} \pi \gamma d\left(1-\mathrm{e}^{-b / B}\right)
$$

where $B \approx 230 \mathrm{~nm}$ is a characteristic thickness, which is a few times the particle roughness value $(30 \pm 2 \mathrm{~nm})$. This behavior is reminiscent of what is observed with liquid capillary bridges. In this latter case the cohesion force increases when increasing the amount of liquid, up to the point where the liquid screens the surface roughness and that a single bridge exists, giving rise to the saturated force given by Eq. 2 [15].

From this expression, we can write the expression for a Bond number, i.e. the ratio of the weight of the particle over the cohesion force, a dimensionless number that will be useful in the following :

$$
B o=\frac{1}{9} \frac{\rho_{G} g d^{2}}{\gamma\left(1-\mathrm{e}^{-b / B}\right)}
$$

The threshold value $B o=1$ gives a critical particle size for which the weight is balanced by the cohesion force. A typical example of $B o \approx 1$ is given by a coating thickness $b=2 \mu \mathrm{m}$ with particles $d=3 \mathrm{~mm}$, as illustrated in figure 1 .

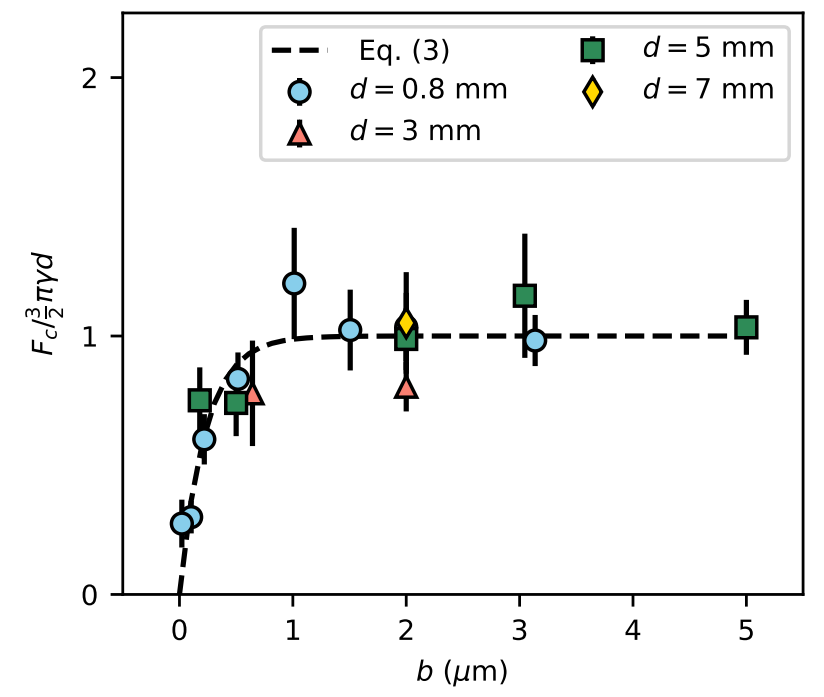

FIG. 8. Cohesion force normalized by $\frac{3}{2} \pi \gamma d$ as a function of the mean PBS layer $b$ for short contact times and for different particle sizes.

\section{BULK BEHAVIOR OF THE CCGM}

The previous sections were dedicated to the measure of the cohesion force between pairs of particles. In this section we discuss the collective behavior of the coated particles in several classical configurations used in the literature for characterizing granular media. The goal is to show that the ability to precisely control the cohesion force between the grains open new perspectives to understand the influence of the inter-particle force in the dynamics of cohesive granular media. This section presents experimental results for the bulk density, for the angle of repose of static piles, and for the onset of the flow of a layer of CCGM resting on an inclined plane.

\section{A. Packing fraction}

In many industrial processes, the bulk density of a granular assembly is a qualitative indicator of the cohesive property of the medium $[30,31]$. The Haussner ratio $\left(H=\rho_{T} / \rho_{B}\right)$ or the Carr index (defined as $100(1-1 / H)$ ) are often used both implying the measurement of the bulk density in two different compaction states: the aerated density $\rho_{B}$ (similar to the random loose packing state) and the tapped density $\rho_{T} /$ (similar to the random close packing). In the following we investigated how the loose packing fraction changes for CCGM batches when varying the PBS coating. The random loose packing fraction $\phi_{r l p}$ was evaluated through mass and volume measurements in a $250 \mathrm{~cm}^{3}$ graduated cylindrical test tube. The results are presented in Fig. 9 for $d=680 \mathrm{~mm}$ particles and for a coating layer thickness varying from 72 


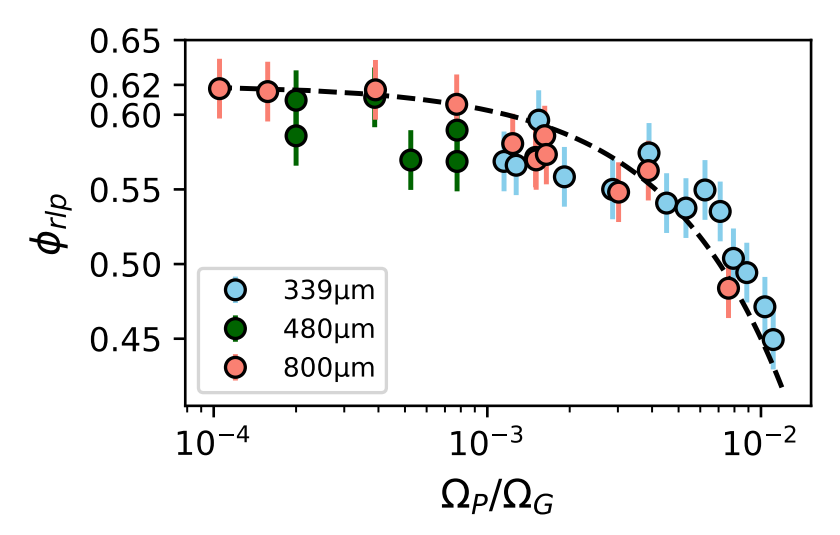

FIG. 9. Random loose packing fraction of various CCGM with different particle sizes and different coating values. The packing fraction decreases with the volume ratio $\Omega_{P} / \Omega_{G}$. The legend indicates the beads diameter.

to $624 \mathrm{~nm}$. The best collapse of experimental data is obtained with a plot of the packing fraction versus the volume ratio $\Omega_{P} / \Omega_{G}$ which is proportional to $b / d$.

For very low values of the coating $\left(\Omega_{P} / \Omega_{G} \approx 10^{-4}\right)$, the packing fraction is equal to the packing fraction of clean and dry glass beads. The packing fraction decreases for an increasing PBS content, and a very low packing fraction $\phi_{r l p} \approx 0.45$ may be reached for a typical $\Omega_{P} / \Omega_{G} \approx 10^{-2}$ value. This can be explained by the existence of large-scale voids and arches in the bulk sustained by strong cohesive links between particles.

\section{B. Angle of repose}

The measure of the slope angle of a granular heap is also a way to emphasize the role of the cohesion in a powder or in a granular material $[14,32-36]$. With our CCGM, static piles were made from a chute flow from a hopper on a $5 \mathrm{~cm}$ diameter rough disc. A side-view camera captured the image of a pile and the angle of repose $\alpha_{r}$ is obtained from image analysis. Examples of images of piles are given in Fig. 10. The value of the repose angle is averaged over 20 measurements. The repose angle is observed to increase when increasing the PBS thickness. Without coating (Fig. 10a), the heap presents a smooth surface with a constant angle. Adding some cohesion gives rise to steeper slopes, and also to abrupt local variations of the local angle as seen illustrated in Fig. 10(b-d).

Fig. 11 shows that the angle $\theta_{r}$ increases with the coating thickness $b$, with a sharp increase for $b \approx 40 \mathrm{~nm}$ and seems to saturate for coatings larger than $50 \mathrm{~nm}$. We compare our results with the repose angle obtained with the crater method using vacuum pump oil coated particles [14, 34] in figure 11. While the heap formation methods are different, the CCGM presents a similar trend. Our experimental setup is limited to low-cohesion

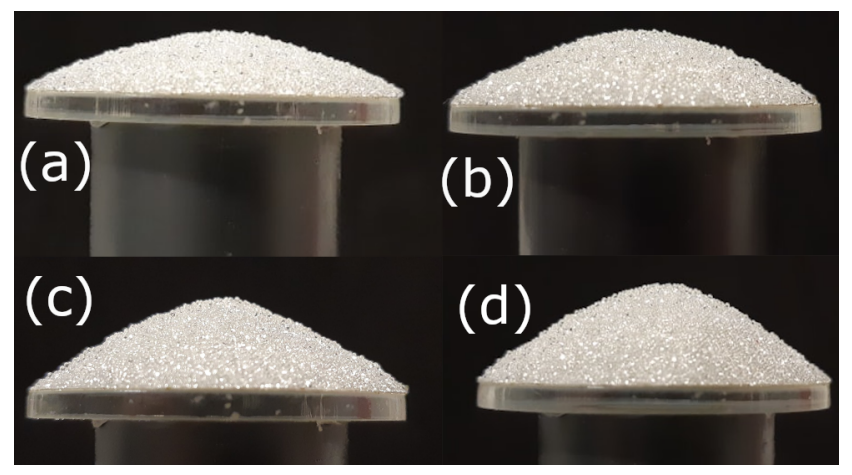

FIG. 10. Images of piles for a CCGM with $d=480 \mu \mathrm{m}$ with increasing PBS coating: (a) no coating, $\alpha_{r}=27.7 \pm 0.8$, (b) $b=31 \mathrm{~nm}, \alpha_{r}=30.1 \pm 0.9$, (c) $b=52 \mathrm{~nm}, \alpha_{r}=40.3 \pm 1.9$, (d) $b=62 \mathrm{~nm}, \alpha_{r}=42.5 \pm 2.2$

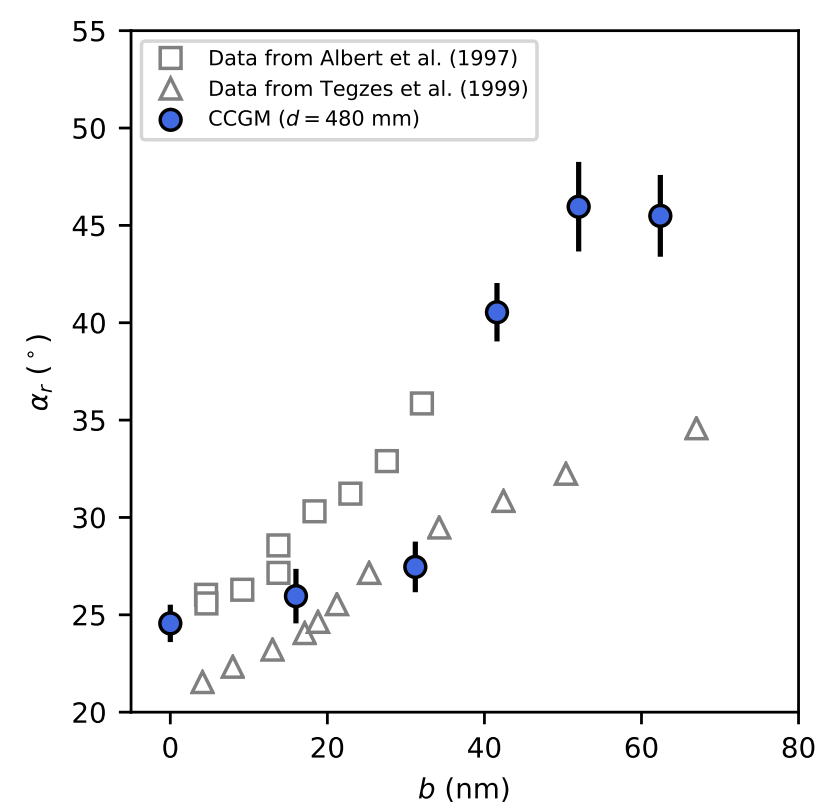

FIG. 11. Heap repose angle for $d=480 \mu \mathrm{m}$ particles and various coatings. Empty symbols are data from the literature with capillary cohesion: $d=800 \mu \mathrm{m}$ [14] (squares) and $d=$ $900 \mu \mathrm{m}$ [34] (triangles).

values because the materials prepared with a large cohesion $(b>100 \mathrm{~nm})$ experience difficulties to flow through the hopper under gravity only.

The heap angle experiment is also a benchmark test to assess the stability and the durability of the CCGM. The first test concerns the stability with temperature since the cohesion is based on a cross-linked polymer. Piles have been prepared with a CCGM stored in controlledtemperature devices. As shown in Fig. 12(a), the repose angle is nearly independent of temperature from $0^{\circ} \mathrm{C}$ to $60^{\circ} \mathrm{C}$. No noticeable difference was found between experiments at $20^{\circ} \mathrm{C}$ and $60^{\circ} \mathrm{C}$. This means that no specific care is needed for experiments at a standard room tem- 

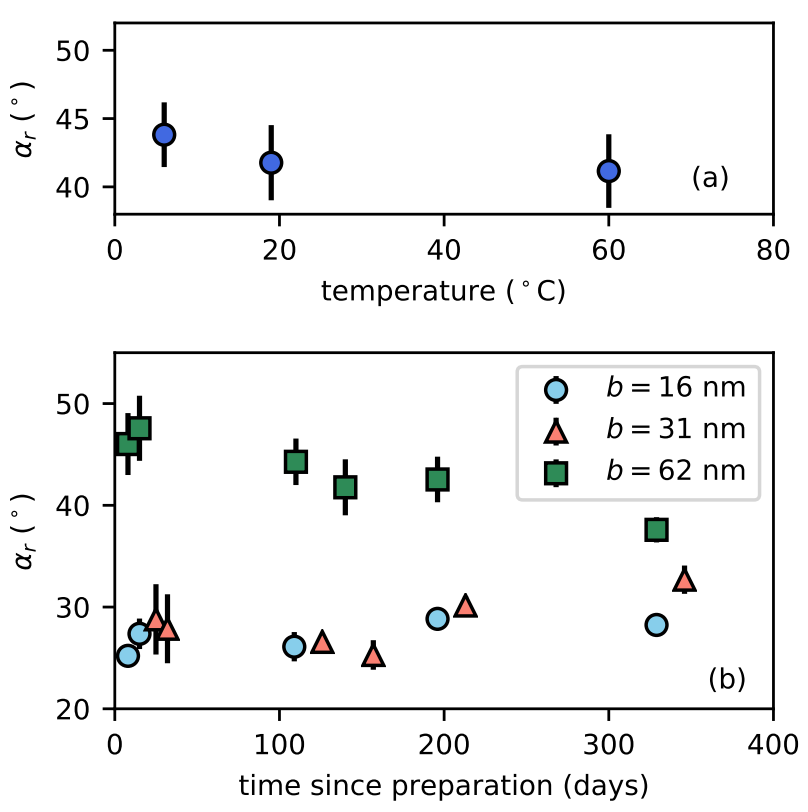

FIG. 12. (a) Effect of the temperature on the repose angle of a CCGM $(d=480 \mu \mathrm{m}, b=62 \mathrm{~nm})$. (b) Heap repose angle $\alpha_{r}$ as a function of the time since the preparation of the CCGM. Experiments were made with $d=480 \mu \mathrm{m}$ particles.

perature.

We have also investigated the stability of the CCGM with time by measuring the heap repose angle for the same batch of particles at different ages. Fig. 12(b) shows the evolution of the repose angle $\alpha_{r}$ for three different materials at different ages from preparation. This plot shows that for thin coatings $(b=16$ or $b=31 \mathrm{~nm})$, the heap angle remains identical even for sample prepared one year ago. For a thicker coating $(b=62 \mathrm{~nm})$, a slow decrease of the repose angle has been observed. Nevertheless, the PBS-coated CCGM seems to be stable for months and large batches can then be prepared before performing large-scale experiments. This CCGM also seems to be unaffected by the moisture content of the ambient air since our experiments were performed without humidity control across different temperature and humidity conditions over a year.

\section{Onset of flow on an inclined plane}

Measuring the onset of flow of a layer of particles lying on a rough inclined bed is another way to investigate the friction and the cohesion of a material. The simplest description of the plasticity of a granular material assumes that the yield stress follows a cohesive Mohr-Coulomb criterion, $\tau_{\text {yield }}=\mu P+\tau_{c}$, where $\mu$ is the friction coefficient, $P$ the confining pressure and $\tau_{c}$ the cohesive stress. Starting from an horizontal plane and increasing progressively the inclination $\theta$, a layer of thickness $h$ will start

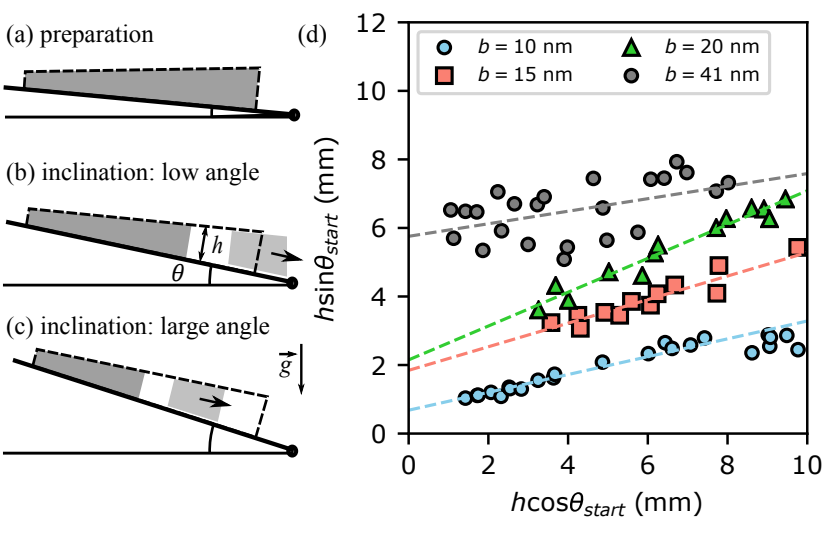

FIG. 13. (a)-(c): Sketch of the inclined plane setup with the variable granular thickness and a progressive inclination. (d): Inclined plane results for $d=202 \mu \mathrm{m}$ CCGM particles with increasing coating thickness. Dashed lines are best fits using Eq. (6).

to flow at a critical angle $\theta_{\text {start }}$ when the shear stress at the base reaches the yield stress value:

$$
\rho_{G} \phi g h \sin \theta_{\text {start }}=\mu \rho_{G} \phi g h \cos \theta_{\text {start }}+\tau_{c}
$$

where $\phi$ is the volume fraction of the layer. This equation can be simplified as

$$
h \sin \theta_{\text {start }}=\mu h \cos \theta_{\text {start }}+h_{c}
$$

where $h_{c}=\tau_{c} /\left(\phi \rho_{G} g\right)$ is a characteristic cohesive length, which represents the maximum thickness of a self-standing vertical layer of granular medium stuck to a rough surface under gravity.

Eq. 6 shows that the cohesion length $h_{c}$ and the friction coefficient $\mu$ can be identified by systematically measuring the critical starting angle $\theta_{\text {start }}$ for different thicknesses $h$. We have conducted such a series of experiments with our model cohesive material. For seek of efficiency, we have not used a uniform layer as initial state, but rather a prismatic deposit, as sketched in Fig. 13(a). With this geometry, it is possible in a single experiment to perform several measurements of $\left(h, \theta_{\text {start }}\right)$.

A typical experiment is conducted as follows. The CCGM is poured on a $20 \times 6 \mathrm{~cm}^{2}$ rectangular rough plate (the roughness is made with CCGM particles glued on a double sided adhesive tape) with two prismatic side walls. The free surface of the deposit is then leveled following the two side walls. The final prismatic volume has a thickness varying linearly from 15 to $25 \mathrm{~mm}$ (see Fig. 13a). The thickness of the granular layer is measured with a laser sheet technique and the angle with a clinometer. Starting from a very low angle of inclination (typically $10^{\circ}$ ), the setup is slowly inclined at a constant rate. A first avalanche occurs at the bottom thick side (Fig. 13b), leaving a thinner and shorter prism. When the angle is further increased, a second avalanche occurs (Fig. 13c), which corresponds to a different $h$, 
and so on. In one experiment, one can then extract the critical angle $\theta_{\text {start }}$ for 4 to 5 different thicknesses. For a single CCGM batch, this experiment is repeated several times. The collected data are then plotted in a ( $\left.h \cos \theta_{\text {start }}, h \sin \theta_{\text {start }}\right)$ plane. According to Eq. 6, a linear fit of experimental data gives the slope $\mu$ and the intercept value $h_{c}$ for a given CCGM. Fig. 13(d) shows the $\left(h \cos \theta_{\text {start }}, h \sin \theta_{\text {start }}\right)$ plot for small glass beads of diameter $d=202 \pm 4 \mu \mathrm{m}$. Results are given for 4 coatings of increasing thickness $b$. Despite some experimental noise, the linear expression (5) fits well the experimental data. Increasing the coating thickness $b$ increases the cohesive length $h_{c}$ (the intercept of the linear fit with the vertical axis), but does not significantly affect the friction coefficient (the slope of the lines). From the measure of $h_{c}$, one can then estimate the cohesive stress $\tau_{c}$.

The stress $\tau_{c}$ is a macroscopic measurement of the cohesion, which can be compared to the inter-particle cohesion force measured in section III. From a dimensional analysis, the scaling relating the cohesive shear stress and the cohesion force is $\tau_{c} d^{2} \propto F_{c}$. Following [17], a theoretical expression based on a mean field approach relates the macroscopic cohesion $\tau_{c}$ to the cohesion force:

$$
\tau_{c}=\frac{3 \mu \phi Z F_{c}}{2 \pi d^{2}},
$$

with $\mu$ the friction coefficient, $\phi$ the volume fraction and $Z$ the averaged coordinance number (number of contacts per particle). Fig. 14 gathers our data for different particle sizes and different PBS coatings and shows a linear trend between $\tau_{c} d^{2}$ and $F_{c}$ although it is not perfect especially for small particles. The presence of other cohesive forces like humidity may explain this discrepancy observed for small particles. Taking the experimental averaged value $\mu=0.4$ along with $\phi=0.6$ and $Z=6$, the theoretical prediction is plotted in Fig. 14 and gives indeed a good estimate of the measured cohesion. The insert of Fig. 14 also shows that the macroscopic friction coefficient $\mu$ seems independent of the coating property of the particles.

\section{CONCLUDING REMARKS AND PERSPECTIVES}

A cohesion-controlled granular material (CCGM) has been designed with glass beads coated by a thin layer of polyborosiloxane polymer. The preparation technique is simple, the resulting CCGM is very robust and can be used several times in various situations to model industrial or natural cohesive granular materials. We have investigated how the inter-particle cohesive contact force varies with the thickness of the coating and with the particle size and have showed that the cohesion force does not depend on the pre-compression force. We also showed that the cohesion force slightly evolves with time and eventually saturates for long time.

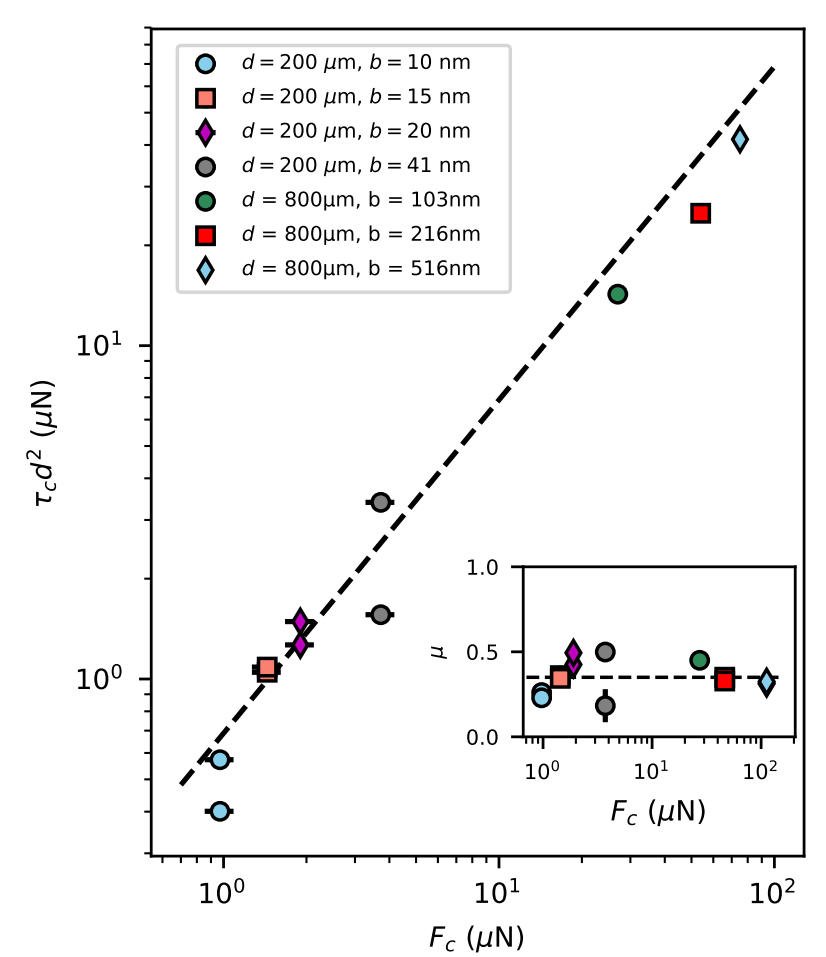

FIG. 14. The macroscopic cohesion force $\tau_{c} d^{2}$ measured from inclined plane experiments as a function of the inter-particle cohesive force $F_{c}$. The dashed line is the prediction from Eq. (7).

Different experimental setups were used to put in evidence the influence of the cohesion on various macroscopic quantities. We have illustrated the effect of the cohesion on the packing fraction of loose samples, on the repose angle of heaps, on the onset of flow of a granular deposit on a rough bed. In all cases we have shown that controlling the inter-particle force is a way to controlled the macroscopic properties. This CCGM seems to be very stable in time and weakly affected by the humidity or the temperature of the laboratory. This is a major advantage compared to previous capillary cohesion techniques based on Newtonian fluids where drainage, evaporation, capillary bridges recombination may occur during an experiment.

In this paper we have restricted our observation to static configurations. However, the possibility to control the cohesion open many perspectives to study also the flow properties of cohesive materials. Configurations such as flows down inclined planes, flows in hoppers and silos, flows in rotating drums, mixing or segregation may also be investigated, with the hope that it will help to better understand the physics beyond the concept of "flowability" of cohesive granular materials. 


\section{ACKNOWLEDGEMENTS}

We thank Inès Basses, Davide Di Giusto and Amina Abbedou for their help during the experiments and
Sandip Mandal for fruitful discussion and improvement of the paper. This work is part of the COPRINT [37] project and was supported by the ANR grant ANR-17CE08-0017.
[1] L. Bocquet, E. Charlaix, S. Ciliberto, and J. Crassous, Moisture-induced ageing ingranular media and the kinetics of capillary condensation, Nature 396, 735 (1998).

[2] N. Mitaraiand F. Nori, Wet granular materials, Advances in physics 55, 1 (2006).

[3] A. Castellanos, The relationship between attractive interparticle forces and bulk behaviour in dry and uncharged fine powders, Advances in Physics 54, 263 (2005).

[4] L. Konopkaand J. Kosek, Discrete element modeling of electrostatic charging of polyethylene powder particles, Journal of electrostatics 87, 150 (2017).

[5] M. V. Antequera, A. M. Ruiz, M. M. Perales, N. M. M. noz, and M. J.-C. Ballesteros, Evaluation of an adequate method of estimating flowability according to powder characteristics, International Journal of Pharmaceutics 103, 155 (1994).

[6] D. Geldart, E. C. Abdullah, A. Hassanpour, L. C. Nwoke, and I. Wouters, Characterization of powder flowability using measurement of angle of repose, China Particuology 4, 104 (2006).

[7] S. Luding, Cohesive, frictional powders: contact models for tension, Granular matter 10, 235 (2008).

[8] D. Howell, I. Aranson, and G. Crabtree, Dynamics of electrostatically-driven granular media. effects of humidity, Physical Review E 63, 050301 (2001).

[9] R. Jones, H. M. Pollock, D. Geldart, and A. Verlinden, Inter-particle forces in cohesive powders studied by afm: effects of relative humidity, particle size and wall adhesion, Powder Technology 132, 196 (2003).

[10] A. W. Alexander, B. Chaudhuri, A. Faqih, F. J. Muzzio, C. Davies, and M. S. Tomassone, Avalanching flow of cohesive powders, Powder Technology 164, 13 (2006).

[11] N. Vandewalle, G. Lumay, F. Ludewig, and J. E. Fiscina, How relative humidity affects random packing experiments, Physical Review E 85, 031309 (2012).

[12] J. Q. Xu, R. P. Zou, and A. B. Yu, Analysis of the packing structure of wet spheres by voronoidelaunay tessellation, Granular matter 9, 455 (2007).

[13] D. J. Hornbaker, R. Albert, I. Albert, A.-L. Barabasi, and P. Schiffer, What keeps sandcastles standing?, Nature 387, 765 (1997).

[14] R. Albert, I. Albert, D. Hornbaker, P. Schiffer, and A.L. Barabasi, Maximum angle of stability in wet and dry spherical granular media, Physical Review E 56, 6271 (1997).

[15] T. C. Halseyand A. J. Levine, How sandcastles fall, Physical Review Letters 80, 3141 (1998).

[16] S. Nowak, A. Samadani, and A. Kudrolli, Maximum angle of stability of a wet granular pile, Nature 1, 50 (2005).

[17] V. Richefeu, M.-S. El Youssoufi, and F. Radjai, Shear strength properties of wet granular materials, Physical Review E 73, 051304 (2006).

[18] P. M. 1. M. Pakpour, M. Habibiand D. Bonn, How to construct the perfect sandcastle, Scientific Reports 2, 549 (2012).
[19] P. C. F. M. llerand D. Bonn, The shear modulus of wet granular matter, Europhysics Letters 80, 38002 (2007).

[20] M. Badetti, A. Fall, and J.-N. Roux, Rheology of wet granular materials in shear flow: experiments and discrete simulations, E3S Web of conferences 9, 14008 (2016).

[21] M. Badetti, A. Fall, F. Chevoir, P. Aimedieu, S. Rodts, and J.-N. Roux, Rheology and microstructure of unsaturated granular materials: Experiments and simulations, Journal of rheology 62, 1175 (2018).

[22] C. D. Willett, M. J. Adams, S. A. Johnson, and J. P. K. Seville, Capillary bridges between two spherical bodies, Langmuir 16, 9396 (2000).

[23] R. Mani, D. Kadau, and H. J. Herrmann, Liquid migration in sheared unsaturated granular media, Granular Matter 15, 447 (2013).

[24] G. Gagneuxand O. Millet, An analytical framework for evaluating the cohesion effects of coalescence between capillary bridges, Granular matter 18, 16 (2016).

[25] A. Jarray, H. Shi, B. Scheper, M. Habibi, and S. Luding, Cohesion-driven mixing and segregation of dry granular media, Nature Scientific Reports 9, 13480 (2019).

[26] J. Modelland S. Thuresson, Material composition and lmethod for its manufacturing, Patent WO2008/020800A1 (2008).

[27] X. Li, D. Zhang, K. Xiang, and G. Huang, Synthesis of polyborosiloxane and its reversible physical crosslinks, RSC Advances 4, 32894 (2014).

[28] T. Kobayashi, T. Tanaka, N. Shimada, and T. Kawaguchi, Dem-cfd analysis of fluidization behavior of geldart group a particles using a dynamic adhesion force model, Powder Technology 248, 143 (2013).

[29] K. Johnson, K. Kendall, and A. D. Roberts, Surface energy and the contact of elastic solids, Proceedings of the royal society of London. A. mathematical and physical sciences 324, 301 (1971).

[30] N. Standish, A. Yu, and Q. He, An experimental study of the packing of a coal heap, Powder Tech. 68, 187 (1991).

[31] A. Hassanpour, C. Hare, and M. Pasha, eds., Powder Flow: Theory, Characterisation and Application (Royal Society of Chemistry, 2019).

[32] S. R. Miles, The relation between the moisture content and the test weight of corn, J. Am. Soc. Agronomy 29, 412 (1937).

[33] E. F. Wolfand H. L. Hohenleiten, Experimental study of the flow of coal in chutes, Trans. Am. Soc. Mech. Eng 67, 585 (1945).

[34] P. Tegzes, R. Albert, M. Paskvan, A.-L. Barabasi, T. Vicsek, and P. Schiffer, Liquid-induced transitions in granular media, Physical Review E 60, 5823 (1999).

[35] A. Samadaniand A. Kudrolli, Angle of repose and segregation in cohesive granular matter, Physical Review E 64, 051301 (2001). 
[36] A. de Ryck, R. Condotta, and J. A. Dodds, Shape of a cohesive granular heap, Powder Technology 157, 72
(2005).

[37] Coprint, http://coprint226940055. wordpress.com. 